\title{
Auslegung des Vorteilsverbots von Art. 33 HMG
}

\author{
Swissmedic äussert sich zum Verbot des Gewährens bzw. Anbietens geld- \\ werter Vorteile gemäss Artikel 33 des Heilmittelgesetzes (HMG) und zum \\ Verhältnis dieser Gesetzesbestimmung zu den Richtlinien «Zusammenarbeit \\ Ärzteschaft-Industrie» der Schweizerischen Akademie der medizinischen \\ Wissenschaften (SAMW).
}

Swissmedic, Schweizerisches Heilmittelinstitut

* Die offizielle Bezeichnung lautet: Bundesgesetz über Arzneimittel und Medizinprodukte.

1 Swissmedic. Zulässigkeit von Rabatten im Rahmen von Artikel 33 Absatz 3 Buchstabe b des Heilmittelgesetzes. Swissmedic Journal. 2003;(11):980-4 www.swissmedic.ch $\rightarrow$ «für Fachpersonen» $\rightarrow$ «Publikationen».

2 Swissmedic. Zum Verbot des Versprechens und Annehmens geldwerter Vorteile gemäss Artikel 33 des Heilmittelgesetzes insb. in Zusammenhang mit der Unterstützung der Weiter- und Fortbildung von Medizinalpersonen durch die Pharmaindustrie. Swissmedic Journal. 2006;(1):20-45. www.swissmedic.ch $\rightarrow$ «für Fachpersonen» $\rightarrow$ «Publikationen».

Korrespondenz:

Swissmedic, Schweizerisches

Heilmittelinstitut

Hallerstrasse 7

Postfach

CH-3000 Bern 9

\section{Auslegung des Vorteilsverbots von Art. 33 HMG durch das Schweizerische Heilmittelinstitut Swissmedic als zuständige Vollzugsbehörde}

Das seit 2002 geltende Heilmittelgesetz (HMG)* verbietet in Artikel 33 das Gewähren, Anbieten oder Versprechen geldwerter Vorteile an Personen, die Arzneimittel verschreiben oder abgeben (insbesondere Ärztinnen und Ärzte sowie Apotheken), oder an Organisationen, die solche Personen beschäftigen (insbesondere Spitäler). Ebenso dürfen diese Personen und Organisationen selber keine solchen Vorteile fordern oder annehmen. Die Swissmedic ist vom Gesetzgeber beauftragt, dieses Vorteilsverbot mittels Verwaltungsmassnahmeverfahren durchzusetzen. Sofern es sich nicht um besonders leichte Verstösse handelt, hat Swissmedic zudem ein Strafverfahren zu eröffnen und gegen die Beteiligten Bussen bis zu 50000 Franken zu verhängen.

Das Vorteilsverbot von Art. 33 HMG und insbesondere dessen Ausnahmebestimmungen (in Art. 33 Abs. 3 HMG) haben in den ersten Jahren seit Inkrafttreten des Heilmittelgesetzes zu etlichen Interpretationsfragen geführt. Einen wesentlichen Teil dieser Fragen hat Swissmedic in zwei Publikationen vom November 2003 und Januar 2006 zuhanden der interessierten Öffentlichkeit geklärt [1, 2]. Darin wurde festgehalten, dass das Vorteilsverbot von Art. 33 HMG bereits dann gilt, wenn ein indirekter (mittelbarer) Zusammenhang zwischen einem geldwerten Vorteil sowie einem oder mehreren bestimmten Arzneimittel(n) oder Gruppen von Arzneimitteln besteht. Dieser Zusammenhang kann demnach aus den Begleitumständen hergeleitet werden: $\mathrm{Er}$ besteht beispielsweise im Verhältnis zwischen einem Pharmaunternehmen sowie Ärztinnen und Ärzten, die aufgrund ihrer Spezialisierung in ihrer täglichen Praxis ein Arzneimittel verschreiben, das von diesem Unternehmen hergestellt oder vertrieben wird. Wenn das Unternehmen diese Ärztinnen und Ärzte nun zu einer Fortbil- dungsveranstaltung einlädt, muss das Vorteilsverbot von Art. 33 HMG beachtet werden. Schliessen sich zum selben Zweck eine Mehrheit von Pharmaunternehmen zusammen, die in direkter Konkurrenz zueinander stehen, d.h. auf dem Markt Arzneimittel für dieselbe medizinische Indikation anbieten, so ist das Vorteilsverbot von Art. $33 \mathrm{HMG}$ jedoch nicht oder nur eingeschränkt anwendbar.

Findet das Vorteilsverbot von Art. 33 HMG auf medizinische Weiter- und Fortbildungsveranstaltungen Anwendung, haben die eingeladenen Ärztinnen und Ärzte in der Regel einen Selbstkostenbeitrag zu leisten, sofern die Veranstaltung länger als einen halben Tag dauert. Die Höhe dieses Selbstkostenbeitrags beträgt in der Regel mindestens ein Drittel (33\%) sämtlicher auf einen Teilnehmer entfallender Kosten; für Medizinalpersonen in Weiterbildung kann er auf ein Fünftel (20\%) der Gesamtkosten reduziert oder unter bestimmten Umständen sogar völlig erlassen werden. Keinen Selbstkostenbeitrag schulden auch Veranstaltungsteilnehmer, die eigene, aktive Beiträge leisten. Das Rahmenprogramm einer Veranstaltung darf sodann nicht mehr als 20\% des finanziellen und zeitlichen Umfangs der gesamten Veranstaltung ausmachen; es muss zudem unmittelbar vor, während oder unmittelbar nach der Veranstaltung angeboten werden. Mitreisende Begleitpersonen haben sämtliche Kosten selber zu tragen. Für Einzelheiten wird auf die Publikation im Swissmedic Journal verwiesen [2].

\section{Bedeutung der SAMW-Richtlinien «Zusammenarbeit Ärzteschaft-Industrie» für die Auslegung des Vorteilsverbots von Art. 33 HMG}

Die Schweizerische Akademie der medizinischen Wissenschaften (SAMW) hat im Jahr 2002 erstmals Empfehlungen zur «Zusammenarbeit Ärzteschaft-Industrie» veröffentlicht; darin werden bezüglich der finanziellen Unterstützung von 
3 SAMW. Zusammenarbeit Ärzteschaft-Industrie. Schweiz Ärztezeitung. 2002;83(41):2165-71.

4 SAMW. Zusammenarbeit Ärzteschaft-Industrie. Schweiz Ärztezeitung. 2006;87(5):177-83.

5 Rüetschi D. Die Medizinischethischen Richtlinien der SAMW aus juristischer Sicht. Schweiz Ärztezeitung. 2004;85(23):1222-5 «Da es sich bei der SAMW um eine Stiftung gemäss Art. 80 ff. ZGB handelt und ihr auch keine hoheitlichen Befugnisse übertragen worden sind, stellen die Richtlinien keine eigentlichen Rechtsnormen dar, sondern lediglich Empfehlungen einer privaten Organisation an ihre Mitglieder.»

6 Siehe dazu [2] speziell S. 28 (Ziff. III.2.2).

7 Demgegenüber können sich Mitarbeitende eines Pharmaunternehmens, das zu einer Veranstaltung einlädt oder diese mitfinanziert, nicht auf die SAMW-Richtlinien berufen. Für sie hat die Swissmedic gewisse Empfehlungen formuliert (vgl. [2] speziell S. 43 [Ziff. IV.3.7]).
Aus-, Weiter- und Fortbildungsveranstaltungen durch die pharmazeutische Industrie sowie durch die Medizinproduktebranche verschiedene Anforderungen formuliert: So wird u.a. nahegelegt, dass Ärztinnen und Ärzte in leitender oder selbständiger Stellung bei Teilnahme an Veranstaltungen, die in Europa stattfinden, einen Selbstkostenbeitrag von mindestens 500 Franken und bei solchen ausserhalb Europas einen Beitrag von mindestens 1000 Franken übernehmen sollten [3]. Diese Empfehlungen wurden 2004 revidiert und Anfang Februar 2006 veröffentlicht [4]. Die Ärztekammer der FMH hat diese Richtlinien in ihrer Sitzung von 19. Mai 2006 in die FMH-Standesordnung aufgenommen.

Die SAMW-Richtlinien zur «Zusammenarbeit Ärzteschaft-Industrie» sind für die Swissmedic als zuständige Behörde eine wichtige Interpretationshilfe bei der Anwendung des Vorteilsverbots von Art. 33 HMG: Sie vermitteln Aufschluss darüber, was die Betroffenen selber für noch zulässig halten; sie verstärken damit das Gerüst staatlicher Regelungen aus berufsethischer Sicht, wodurch sie zum gesetzeskonformen Verhalten aller Beteiligten beitragen. Die SAMW-Richtlinien und insbesondere die ihr vorangegangenen Empfehlungen aus dem Jahr 2002 sind deshalb als um so wertvoller einzustufen, weil sie bereits in einer Zeit Anhaltspunkte zur Auslegung von Art. 33 HMG gegeben haben, als Swissmedic noch keine ihrer obenerwähnten Interpretationen veröffentlicht hatte.

Es gilt jedoch festzuhalten, dass die SAMWRichtlinien zur «Zusammenarbeit ÄrzteschaftIndustrie» - gleich wie alle übrigen Richtlinien, Standesregeln und Codes of Conduct von Branchenverbänden und -organisationen - für Swissmedic nicht verbindlich sind [5]. Swissmedic muss insbesondere dann einen strengeren Massstab anwenden, als es die SAMW-Richtlinien tun, wenn sie in Auslegung des Heilmittelgesetzes und seiner Ausführungsverordnungen zur Auffassung gelangt, das Gesetz mache andere, strengere Vorgaben. Swissmedic handelt dabei im Rahmen rechtsstaatlich vorgesehener Verfahren; ihre Entscheidungen können stets mit Rechtsmitteln angefochten und damit einer gerichtlichen Beurteilung zugeführt werden.

Ferner gilt es darauf hinzuweisen, dass der Anwendungsbereich der SAMW-Richtlinien umfassender ist als derjenige des Vorteilsverbots von Art. $33 \mathrm{HMG}$, indem diese Richtlinien auch die Beziehungen der Ärzteschaft zur Medizinproduktebranche erfassen. Demgegenüber beschränkt sich das Verbot von Art. 33 HMG auf Human- und Tierarzneimittel und ist nur in Ausnahmefällen auch auf Medizinprodukte anwendbar [6].

Für Ärztinnen und Ärzte, die von einem Pharmaunternehmen eine Einladung zur Teilnahme an einer Weiter- und Fortbildungsveranstaltung erhalten, kann es - weil sie die Gesamtkosten der Veranstaltung nicht kennen - schwierig sein, im voraus abzuschätzen, ob der verlangte Selbstkostenbeitrag genügend hoch ist, damit sie mit ihrer Teilnahme das Vorteilsverbot von Art. 33 HMG nicht verletzen. Ebenso kann es unter Umständen Mühe bereiten zu erkennen, ob ein Rahmenprogramm einer Veranstaltung mehr als die noch zulässigen $20 \%$ des Gesamtaufwands für die Veranstaltung ausmacht.

Swissmedic trägt diesem Umstand Rechnung, indem sie die in den SAMW-Richtlinien aufgestellten Kriterien für die Teilnahme an Weiterund Fortbildungsveranstaltungen als Mindestanforderungen betrachtet, bei deren Einhaltung gegen die teilnehmenden Ärztinnen und Ärzte in der Regel kein Verwaltungsmassnahme- oder -strafverfahren eröffnet wird [7]. Sofern jedoch offensichtlich ist, dass die verlangten Selbstkostenbeiträge zu niedrig sind, wird ein Verfahren auch dann eröffnet, wenn die in den SAMWRichtlinien aufgestellten Anforderungen eingehalten wurden.

Es besteht zudem die Möglichkeit, vorgängig an die Swissmedic zu gelangen, um von ihr beurteilen zu lassen, ob bzw. unter welchen Voraussetzungen die Teilnahme an einer Veranstaltung, zu der die Pharmaindustrie einlädt oder die von ihr unterstützt wird, mit dem Vorteilsverbot von Art. 33 HMG vereinbar ist. 